\title{
$h$-Stability of Dynamic Equations on Time Scales with Nonregressivity
}

\author{
Sung Kyu Choi, ${ }^{1}$ Yoon Hoe Goo, ${ }^{2}$ and Namjip Koo ${ }^{1}$ \\ ${ }^{1}$ Department of Mathematics, Chungnam National University, Daejeon 305-764, South Korea \\ 2 Department of Mathematics, Hanseo University, Seosan 352-820, South Korea
}

Correspondence should be addressed to Namjip Koo, njkoo@math.cnu.ac.kr

Received 25 March 2008; Accepted 31 May 2008

Recommended by Allan Peterson

We study the $h$-stability of dynamic equations on time scales, without the regressivity condition on the right-hand side of dynamic equations. This means that we can include noninvertible difference equations into our results.

Copyright (C) 2008 Sung Kyu Choi et al. This is an open access article distributed under the Creative Commons Attribution License, which permits unrestricted use, distribution, and reproduction in any medium, provided the original work is properly cited.

\section{Introduction}

The theory of calculus on time scales, which has recently received a lot of attention, was created by Hilger [1] in order to unify the theories of differential equations and of difference equations and in order to extend those theories to other kinds of the so-called "dynamic equations." The two main features of the calculus on time scales are unification and extension of continuous and discrete analysis.

Time scale calculus is especially useful when studying systems with discrete and continuous elements in their domains, such as an insect population that is continuous for parts of the year and discrete for other parts of the year.

The calculus on time scales and dynamic equations on time scales have applications in any field that requires simultaneous modeling of continuous and discrete processes, because they bridge the divide between continuous and discrete aspects of processes. The applications include insect population models, epidemic models, neural networks, and heat transfer. Foundational definitions and results from the time scale calculus appear in an excellent introductory text by Bohner and Peterson [2].

In [3], we extended the concept of $h$-stability introduced by Pinto [4] to dynamic equations on time scales. The notion of $h$-stability is quite flexible because it includes the 
classical notions of uniform or exponential stability within one common framework. For the detailed results of $h$-stability for differential and difference systems, see [4-7].

Regressivity plays a crucial role in developing the fundamental theory of linear dynamic equations. Consider the scalar dynamic equation on time scale $\mathbb{T}$ :

$$
x^{\Delta}(t)=a(t) x(t) .
$$

Equation (1.1) is said to be regressive if $a(t)$ satisfies

$$
1+a(t) \mu(t) \neq 0
$$

for all $t \in \mathbb{T}^{\kappa}$. A system is nonregressive if it is not regressive. When we consider the generalized time scale exponential function, we need the concept of regressiveness since the exponential function $e_{a}\left(t, t_{0}\right)$ is defined only for $a(t)$ satisfying condition (1.2). The continuous dynamical systems (e.g., ordinary differential equations) are always regressive since $\mathbb{T}=\mathbb{R}$ has the graininess function $\mu(t) \equiv 0$. However, nonregressivity is always a possibility in discrete dynamical systems (e.g., difference equations), where the underlying domain consists of a mixture of discrete and continuous parts. In fact, if there is even one point in $\mathbb{T}$ with nonzero graininess, then nonregressivity is possible [8].

In this paper, we investigate the $h$-stability for dynamic equations on time scales, with the nonregressivity condition. Thus, we improve some results in [3].

\section{Calculus on time scales}

We mention without proof several foundational definitions and theorems, as well as a general introduction to the theory of time scales in an excellent introductory text by Bohner and Peterson [2].

Definition 2.1. A time scale $\mathbb{T}$ is any nonempty closed subset of the real numbers $\mathbb{R}$.

One assumes throughout that $\mathbb{T}$ has the topology that it inherits from the standard topology on $\mathbb{R}$.

It is also assumed throughout that in $\mathbb{T}$ the interval $[a, b]$ means the set $\{t \in \mathbb{T}: a \leq t \leq b\}$ for the points $a<b$ in $\mathbb{T}$. Since a time scale may or may not be connected, one needs the following concept of jump operators.

Definition 2.2. The functions $\sigma, \rho: \mathbb{T} \rightarrow \mathbb{T}$ defined by

$$
\begin{aligned}
& \sigma(t)=\inf \{s \in \mathbb{T}: s>t\}, \\
& \rho(t)=\sup \{s \in \mathbb{T}: s<t\}
\end{aligned}
$$

are called the jump operators.

The jump operators $\sigma$ and $\rho$ allow the classification of points in $\mathbb{T}$ in the following way.

Definition 2.3. A nonmaximal element $t \in \mathbb{T}$ is said to be right-dense if $\sigma(t)=t$, and right-scattered if $\sigma(t)>t$. Also, a nonminimal element $t \in \mathbb{T}$ is called left-dense if $\rho(t)=t$, and left-scattered if $\rho(t)<t$. 
Definition 2.4. The function $\mu: \mathbb{T} \rightarrow \mathbb{R}_{+}$defined by $\mu(t)=\sigma(t)-t$ is called the graininess function.

If $\mathbb{T}$ has a left-scattered maximum $m$, then $\mathbb{T}^{\kappa}=\mathbb{T}-\{m\}$. Otherwise $\mathbb{T}^{\kappa}=\mathbb{T}$.

Definition 2.5. A function $f: \mathbb{T} \rightarrow \mathbb{R}$ is called differentiable at $t \in \mathbb{T}^{\mathcal{K}}$ with (delta) derivative $f^{\Delta}(t) \in \mathbb{R}$ if given $\varepsilon>0$ there exists a neighborhood $U$ of $t$ such that, for all $s \in U$,

$$
\left|f^{\sigma}(t)-f(s)-f^{\Delta}(t)[\sigma(t)-s]\right| \leq \varepsilon|\sigma(t)-s|,
$$

where $f^{\sigma}=f \circ \sigma$. If $f$ is delta differentiable for every $t \in \mathbb{T}^{\kappa}$, then $f: \mathbb{T} \rightarrow \mathbb{R}$ is called delta differentiable on $\mathbb{T}^{\kappa}$.

Some basic properties of delta derivatives are as follows $[9,10]$.

(i) If $f$ is differentiable at $t \in \mathbb{T}^{\kappa}$, then

$$
f^{\sigma}(t)=f(t)+\mu(t) f^{\Delta}(t)
$$

(ii) If both $f$ and $g$ are differentiable at $t \in \mathbb{T}^{\kappa}$, then the product $f g$ is also differentiable at $t \in \mathbb{T}^{\mathcal{K}}$ with

$$
(f g)^{\Delta}(t)=f^{\Delta}(t) g(t)+f^{\sigma}(t) g^{\Delta}(t)=f(t) g^{\Delta}(t)+f^{\Delta}(t) g^{\sigma}(t) .
$$

Definition 2.6. The function $f: \mathbb{T} \rightarrow \mathbb{R}$ is said to be $r d$-continuous (denoted by $f \in C_{\mathrm{rd}}(\mathbb{T}, \mathbb{R})$ ) if

(i) $f$ is continuous at every right-dense point $t \in \mathbb{T}$,

(ii) $\lim _{s \rightarrow t^{-}} f(s)$ exists and is finite at every left-dense point $t \in \mathbb{T}$.

Definition 2.7. Let $f \in C_{\mathrm{rd}}(\mathbb{T}, \mathbb{R})$. The function $g: \mathbb{T} \rightarrow \mathbb{R}$ is called the antiderivative of $f$ on $\mathbb{T}$ if it is differentiable on $\mathbb{T}$ and satisfies $g^{\Delta}(t)=f(t)$ for $t \in \mathbb{T}^{\kappa}$. In this case, one defines

$$
\int_{a}^{t} f(s) \Delta s=g(t)-g(a), \quad t \in \mathbb{T} .
$$

Some basic properties of delta integral are as follows $[2,9,10]$.

Let $f, g: \mathbb{T} \rightarrow \mathbb{R}$ be rd-continuous.

(i) If $r, s \in \mathbb{T}, \alpha, \beta \in \mathbb{R}$, then

$$
\int_{r}^{s}[\alpha f(t)+\beta g(t)] \Delta t=\alpha \int_{r}^{s} f(t) \Delta t+\beta \int_{r}^{s} g(t) \Delta t
$$

(ii) If $t \in \mathbb{T}^{\kappa}$, then

$$
\int_{t}^{\sigma(t)} f(\tau) \Delta \tau=\mu(t) f(t)
$$


(iii) If $\mathbb{T}=\mathbb{R}$ and $a, b \in \mathbb{T}$, then

$$
\int_{a}^{b} f(t) \Delta t=\int_{a}^{b} f(t) d t
$$

where the integral on the right is the usual Riemann integral from calculus.

(iv) If $[a, b]$ consists of only isolated points, then

$$
\int_{a}^{b} f(t) \Delta t= \begin{cases}\sum_{t \in[a, b)} \mu(t) f(t) & \text { if } a<b, \\ 0 & \text { if } a=b, \\ -\sum_{t \in[b, a)} \mu(t) f(t) & \text { if } a>b .\end{cases}
$$

(v) If $\mathbb{T}=\delta \mathbb{Z}$, where $\delta>0$, then

$$
\int_{a}^{b} f(t) \Delta t= \begin{cases}\sum_{k=a / \delta}^{b / \delta-1} f(k \delta) \delta & \text { if } a<b \\ 0 & \text { if } a=b \\ -\sum_{k=b / \delta}^{a / \delta-1} f(k \delta) \delta & \text { if } a>b .\end{cases}
$$

\section{3. $h$-stability}

Let $M_{n}(\mathbb{R})$ be the set of all $n \times n$ matrices over $\mathbb{R}$ and the class of all rd-continuous operators $A: \mathbb{T} \rightarrow M_{n}(\mathbb{R})$ denoted by

$$
C_{\mathrm{rd}}\left(\mathbb{T}, M_{n}(\mathbb{R})\right) .
$$

We consider the linear homogeneous dynamic system

$$
x^{\Delta}=A(t) x, \quad x\left(t_{0}\right)=x_{0},
$$

where $A \in C_{\mathrm{rd}}\left(\mathbb{T}, M_{n}(\mathbb{R})\right)$.

Definition 3.1 (see [8]). System (3.2) is said to be regressive if

$$
\operatorname{det}[I+\mu(t) A(t)] \neq 0
$$

for all $t \in \mathbb{T}^{\kappa}$, where $I$ denotes the $n \times n$ identity matrix.

It turns out that condition (3.3) is equivalent to having all of the eigenvalues of $A(t)$ regressive in the sense of (1.2) [8].

The norm of an $n \times n$ matrix $A$ is defined to be

$$
|A|=\max _{1 \leq j \leq n}\left|A^{j}\right|,
$$

where $A^{j}$ is the $j$ th column of $A$.

We recall the notion of the transition matrix of the linear dynamic systems without regressivity. 
Definition 3.2 (see [11, Definition 1.3.5]). Let $\tau \in \mathbb{T}$ and $A \in C_{\mathrm{rd}}\left(\mathbb{T}, M_{n}(\mathbb{R})\right.$ ), and assume that $\Phi_{A}:\{(t, \tau) \in \mathbb{T} \times \mathbb{T}: \tau \leq t\} \rightarrow M_{n}(\mathbb{R})$ is an operator with $\Phi_{A}(\tau, \tau)=I$ for all $\tau \in \mathbb{T}$. Then, the unique solution $\Phi_{A}$ of the IVP

$$
X^{\Delta}(t)=A(t) X(t), \quad X(\tau)=I
$$

is called the transition matrix of (3.2).

Definition 3.3 (see [2, Definition 5.18]). Let $\tau \in \mathbb{T}$ and assume that $A \in C_{\mathrm{rd}}\left(\mathbb{T}, M_{n}(\mathbb{R})\right)$ is regressive. The unique solution $\Phi_{A}(t, \tau)$ of IVP (3.5) is called the matrix exponential function of (3.2).

Note that the solution of (3.2) through $\left(t_{0}, x_{0}\right)$ can be represented as $x(t)=x\left(t, t_{0}, x_{0}\right)=$ $\Phi_{A}\left(t, t_{0}\right) x\left(t_{0}\right)$. The transition operator has the following properties.

Lemma 3.4 (see [11, Theorem 1.3.9]). If $A \in C_{\mathrm{rd}}\left(\mathbb{T}^{\kappa}, M_{n}(\mathbb{R})\right.$ ), then there exists the transition matrix $\Phi_{A}(t, \tau)$ of (3.2) which satisfies the following properties:

(i) $\Phi_{A}:\{(t, \tau) \in \mathbb{T} \times \mathbb{T}: \tau \leq t\} \rightarrow M_{n}(\mathbb{R})$ with $\Phi_{A}(\tau, \tau)=$ I for all $\tau \in \mathbb{T}$;

(ii) $\Phi_{A}(t, \tau)=\Phi_{A}(t, s) \Phi_{A}(s, \tau)$ for all $\tau \leq s \leq t$;

(iii)

$$
\begin{aligned}
& \Phi_{A}^{\Delta_{t}}(t, \tau)=A(t) \Phi_{A}(t, \tau) \quad \forall \tau \leq t \\
& \Phi_{A}^{\Delta_{\tau}}(t, \tau)=-\Phi_{A}(t, \sigma(\tau)) A(\tau) \quad \forall \sigma(\tau) \leq t
\end{aligned}
$$

(iv)

$$
\Phi_{A}(\sigma(\tau), \tau)=I+\mu(\tau) A(\tau) \quad \forall \tau \in \mathbb{T}^{\kappa}
$$

if $A \in C_{\mathrm{rd}}\left(\mathbb{T}^{\kappa}, M_{n}(\mathbb{R})\right)$ is regressive, that is, $(I+\mu(t) A(t))$ is invertible for all $t \in \mathbb{T}^{\kappa}$, then $\Phi_{A}: \mathbb{T} \times \mathbb{T} \rightarrow M_{n}(\mathbb{R})$ satisfies (ii) and (iii) for all $t, \tau \in \mathbb{T}$;

(v) $\Phi_{A}(t, \tau)$ is invertible in $M_{n}(\mathbb{R})$ with $\Phi_{A}(t, \tau)^{-1}=\Phi_{A}(\tau, t)$ for all $t, \tau \in \mathbb{T}$.

Assume throughout that $\mathbb{T}$ is unbounded above.

We recall the definitions about the various types of stability for the solutions of (3.2).

Definition 3.5. The solution $x=0$ of (3.2) is said to be stable if to any pair of numbers $t_{0}, \varepsilon>0$, there exists a $\delta=\delta\left(t_{0}, \varepsilon\right)>0$ such that, for any solution $x\left(t, t_{0}, x_{0}\right)$ of (3.2), the inequality $\left|x_{0}\right| \leq \delta$ implies $|x(t)|<\varepsilon$ for all $t \geq t_{0} \in \mathbb{T}$. A system is said to be stable if all of its solutions are stable.

Definition 3.6. The solution $x=0$ of (3.2) is said to be uniformly stable if it is stable and $\delta$ does not depend on $t_{0}$.

Definition 3.7. The solution $x=0$ of (3.2) is said to be asymptotically stable if it is stable and if there exists a $\delta_{0}>0$ such that $\left|x_{0}\right| \leq \delta_{0}$ implies $|x(t)| \rightarrow 0$ as $t \rightarrow \infty$. 
Pinto [4] introduced the notion of $h$-stability which is an extension of the notions of exponential stability and uniform Lipschitz stability.

Definition 3.8. System (3.2) is said to be

(i) an $h$-system if there exist a positive function $h: \mathbb{T} \rightarrow \mathbb{R}$ and a constant $c \geq 1$ such that

$$
\left|x\left(t, t_{0}, x_{0}\right)\right| \leq c\left|x_{0}\right| h(t) h\left(t_{0}\right)^{-1}, \quad t \geq t_{0},
$$

for $\left|x_{0}\right|$ small enough (here $\left.h(t)^{-1}=1 / h(t)\right)$;

(ii) $h$-stable if system (3.2) is an $h$-system and the function $h$ is bounded.

Remark 3.9. If $h(t)=e^{-t}$, then $h$-stability coincides with exponential stability, and if $h(t)$ is constant, then we have uniform Lipschitz stability.

Example 3.10. A linear dynamic system on time scale $\mathbb{T}$ with $\mu(t) \leq 1 / 2$,

$$
x^{\Delta}(t)=\left[\begin{array}{cc}
-2 & 1 \\
-1 & -\sin t-2
\end{array}\right] x(t),
$$

is $h$-stable [3].

For the various definitions of stability, we refer to [12] and we obtain the following possible implications for system (3.2) among the various types of stability:

$h$-stability $\Longrightarrow$ uniform exponential stability $\Longrightarrow$ uniform Lipschitz stability $\Longrightarrow$ uniform stability

as in [6]. The above implications can be proved by the characterization due to Pinto [7, Lemma 1] for the case $\mathbb{T}=\mathbb{R}$, in terms of the transition matrix for system (3.2).

Now, we consider the linear dynamic system (3.2) without the regressivity condition.

Firstly, we show that stability for solutions of (3.2) is equivalent to boundedness of solutions.

Theorem 3.11. All solutions of (3.2) are stable if and only if they are bounded for all $t \geq t_{0} \in \mathbb{T}$.

Proof. Suppose that the solution $x=0$ of (3.2) is stable. Then, given any $\varepsilon>0$, there exists a $\delta>0$ such that $\left|x_{0}\right|<\delta$ implies $\left|x\left(t, t_{0}, x_{0}\right)\right|<\varepsilon$. However, $\left|x\left(t, t_{0}, x_{0}\right)\right|=\left|\Phi_{A}\left(t, t_{0}\right) x_{0}\right|<\varepsilon$ for all $t \geq t_{0} \in \mathbb{T}$. Now, let $x_{0}$ be a vector $(\delta / 2) e^{j}$ for $j=1,2, \ldots, n$. Then,

$$
\left|\Phi_{A}\left(t, t_{0}\right) x_{0}\right|=\left|x^{j}\left(t, t_{0}\right)\right| \frac{\delta}{2}<\varepsilon, \quad t \geq t_{0}, j=1, \ldots, n,
$$

where $x^{j}\left(t, t_{0}\right)$ is the $j$ th column of $\Phi_{A}\left(t, t_{0}\right)$. Thus,

$$
\left|\Phi_{A}\left(t, t_{0}\right)\right|=\max _{1 \leq j \leq n}\left|x^{j}\left(t, t_{0}\right)\right|<\frac{2 \varepsilon}{\delta}, \quad t \geq t_{0} .
$$

Consequently, for any solution $x\left(t, t_{0}, x_{0}\right)$ of (3.2),

$$
\left|x\left(t, t_{0}, x_{0}\right)\right|=\left|\Phi_{A}\left(t, t_{0}\right) x_{0}\right|<\frac{2 \varepsilon}{\delta}\left|x_{0}\right|=M\left|x_{0}\right|, \quad t \geq t_{0},
$$

where $M=2 \varepsilon / \delta$. That is, all solutions of (3.2) are bounded.

Similarly, we can prove the converse. 
In [12, Theorem 2.1], DaCunha obtained the characterization of uniform stability for the regressive system (3.2). Also, we have the same characterization for the nonregressive system (3.2) in what follows.

Theorem 3.12. Equation (3.2) is uniformly stable if and only if there exists a constant $\gamma>0$ such that

$$
\left|\Phi_{A}\left(t, t_{0}\right)\right| \leq \gamma, \quad t \geq t_{0} \in \mathbb{T},
$$

where $\Phi_{A}\left(t, t_{0}\right)$ is a transition matrix of (3.2).

Proof. Suppose that (3.2) is uniformly stable. Then, for any given $\varepsilon>0$, there exists a $\delta=\delta(\varepsilon)>$ 0 such that $t_{0} \leq t_{1} \in \mathbb{T}$ and $\left|x\left(t_{1}\right)\right|<\delta$ imply $|x(t)|<\varepsilon$ for all $t \geq t_{1} \in \mathbb{T}$. Thus,

$$
\left|\Phi_{A}\left(t, t_{1}\right) x\left(t_{1}\right)\right|<\varepsilon \quad \forall t \geq t_{1} \geq t_{0} \in \mathbb{T} .
$$

Since $x\left(t_{1}\right)=\Phi_{A}\left(t_{1}, t_{0}\right) x_{0}$ can be selected for any $t_{0}$ and $t_{1} \geq t_{0}$, let $x\left(t_{0}\right)$ be a vector $(\delta / 2) e^{j}$ for each $j=1,2, \ldots, n$. Then,

$$
\left|\Phi_{A}\left(t, t_{1}\right) \Phi_{A}\left(t_{1}, t_{0}\right) x_{0}\right|=\left|\Phi_{A}\left(t, t_{0}\right) x_{0}\right|=\left|x^{j}\left(t, t_{0}\right)\right| \frac{\delta}{2}<\varepsilon, \quad t \geq t_{1} \geq t_{0}
$$

where $x^{j}\left(t, t_{0}\right)$ is the $j$ th column of $\Phi_{A}\left(t, t_{0}\right)$. Thus,

$$
\left|\Phi_{A}\left(t, t_{0}\right)\right|=\max _{1 \leq j \leq n}\left|x^{j}\left(t, t_{0}\right)\right|<\gamma, \quad t \geq t_{0}
$$

where $\gamma=2 \varepsilon / \delta$. We see that $\left|\Phi_{A}\left(t, t_{0}\right)\right| \leq \gamma$ for all $t, t_{0} \in \mathbb{T}$ with $t \geq t_{0}$.

Conversely, suppose that there exists a $\gamma>0$ such that $\left|\Phi_{A}\left(t, t_{0}\right)\right| \leq \gamma$ for all $t, t_{0} \in \mathbb{T}$ with $t \geq t_{0}$. For any $t_{0}$ and $x\left(t_{0}\right)=x_{0}$, the solution of (3.2) satisfies

$$
|x(t)|=\left|\Phi_{A}\left(t, t_{0}\right) x_{0}\right| \leq\left|\Phi_{A}\left(t, t_{0}\right)\right|\left|x_{0}\right| \leq \gamma\left|x_{0}\right|, \quad t \geq t_{0} .
$$

Thus, uniform stability of (3.2) is established. follows.

Pinto [4] gave the characterization of $h$-system. We obtain the time scale version in what

Theorem 3.13. Equation (3.2) is an h-system if and only if there exist a positive function $h$ defined on $\mathbb{T}$ and a constant $c \geq 1$ such that

$$
\left|\Phi_{A}\left(t, t_{0}\right)\right| \leq \operatorname{ch}(t) h\left(t_{0}\right)^{-1}, \quad t \geq t_{0} \in \mathbb{T},
$$

where $\Phi_{A}\left(t, t_{0}\right)$ is a transition matrix of (3.2).

Proof. Suppose that (3.2) is an $h$-system. Let $x_{0}$ be a vector $(\delta / 2) e^{j}$ for $j=1, \ldots, n$. Then, we have

$$
\left|x\left(t, t_{0}, x_{0}\right)\right|=\left|\Phi_{A}\left(t, t_{0}\right) x_{0}\right|=\left|x^{j}\left(t, t_{0}\right)\right| \frac{\delta}{2} \leq\left|x_{0}\right| \operatorname{ch}(t) h\left(t_{0}\right)^{-1}, \quad t \geq t_{0}, j=1,2, \ldots, n,
$$

where $x^{j}\left(t, t_{0}\right)$ is the $j$ th column of $\Phi_{A}\left(t, t_{0}\right)$. 
Thus, we obtain

$$
\left|\Phi_{A}\left(t, t_{0}\right)\right| \leq \frac{2}{\delta}\left|x_{0}\right| \operatorname{ch}(t) h\left(t_{0}\right)^{-1}=\operatorname{ch}(t) h\left(t_{0}\right)^{-1}, \quad t \geq t_{0}
$$

Conversely, we have

$$
\left|x\left(t, t_{0}, x_{0}\right)\right|=\left|\Phi_{A}\left(t, t_{0}\right) x_{0}\right| \leq\left|\Phi_{A}\left(t, t_{0}\right)\right|\left|x_{0}\right| \leq \operatorname{ch}(t) h\left(t_{0}\right)^{-1}\left|x_{0}\right|, \quad t \geq t_{0}
$$

Hence, (3.2) is an $h$-system.

Remark 3.14. Theorems 3.11, 3.12, and 3.13 also hold for the linear dynamic systems with regressivity.

Now, we study the $h$-stability of the nonlinear perturbed dynamic system without the regressivity condition on the right-hand side of the equation via Gronwall's inequality and Bihari's inequality on time scales.

We consider the perturbed systems of (3.2):

$$
x^{\Delta}=A(t) x+F(t) x, \quad x\left(t_{0}\right)=x_{0},
$$

where $A, F \in C_{\mathrm{rd}}\left(\mathbb{T}, M_{n}(\mathbb{R})\right)$.

We can obtain the following variation-of-constants formula [11].

Lemma 3.15 (see [11]). The solution $x\left(t, t_{0}, x_{0}\right)$ of system (3.24)

$$
x^{\Delta}(t)=A(t) x(t)+g(t, x), \quad t \in \mathbb{T},
$$

where $A \in C_{\mathrm{rd}}\left(\mathbb{T}, M_{n}(\mathbb{R})\right)$ and $g: \mathbb{T} \times \mathbb{R}^{n} \rightarrow \mathbb{R}^{n}$ is $r d$-continuous in the first argument with $g(t, 0)=0$, with the initial value $x\left(t_{0}\right)=x_{0}$ is given by

$$
x\left(t, t_{0}, x_{0}\right)=\Phi_{A}\left(t, t_{0}\right) x_{0}+\int_{t_{0}}^{t} \Phi_{A}(t, \sigma(s)) g(s, x(s)) \Delta s, \quad t \geq t_{0},
$$

where $\Phi_{A}\left(t, t_{0}\right)$ is a transition matrix of (3.2).

Theorem 3.16. Suppose that (3.2) is h-stable. Then, (3.23) is h-stable if there exists a positive constant $\beta$ such that for all $t_{0} \in \mathbb{T}$,

$$
\int_{t_{0}}^{\infty} \frac{h(s)}{h(\sigma(s))}|F(s)| \Delta s \leq \beta
$$

Proof. Since (3.2) is $h$-stable, there exist a constant $c \geq 1$ and a positive bounded function $h(t)$ such that

$$
\left|\Phi_{A}\left(t, t_{0}\right)\right| \leq \operatorname{ch}(t) h\left(t_{0}\right)^{-1}
$$


for all $t \geq t_{0} \in \mathbb{T}$. By Lemma 3.15, the solution $x(t)$ of (3.23) satisfies

$$
x(t)=\Phi_{A}\left(t, t_{0}\right) x_{0}+\int_{t_{0}}^{t} \Phi_{A}(t, \sigma(s)) F(s) x(s) \Delta s, \quad t \geq t_{0},
$$

where $\Phi_{A}\left(t, t_{0}\right)$ is a transition matrix of (3.2).

By taking the norms of both sides of (3.28), we have

$$
|x(t)| \leq \operatorname{ch}(t) h\left(t_{0}\right)^{-1}\left|x_{0}\right|+c \int_{t_{0}}^{t} h(t) h(\sigma(s))^{-1}|F(s)||x(s)| \Delta s, \quad t \geq t_{0} .
$$

Dividing by $h(t)>0$ on both sides,

$$
\frac{|x(t)|}{h(t)} \leq c \frac{\left|x_{0}\right|}{h\left(t_{0}\right)}+c \int_{t_{0}}^{t} \frac{h(s)}{h(\sigma(s))}|F(s)| \frac{|x(s)|}{h(s)} \Delta s, \quad t \geq t_{0} .
$$

In view of Gronwall's inequality on time scales in [9], we obtain

$$
\begin{aligned}
\frac{|x(t)|}{h(t)} & \leq c \frac{\left|x_{0}\right|}{h\left(t_{0}\right)} e_{c(h(s) / h(\sigma(s)))|F(s)|}\left(t, t_{0}\right) \\
& = \begin{cases}c \frac{\left|x_{0}\right|}{h\left(t_{0}\right)} \exp \left(\int_{t_{0}}^{t} \frac{\log (1+\mu(s) c(h(s) / h(\sigma(s)))|F(s)|)}{\mu(s)} \Delta s\right) & \text { if } \mu \neq 0 \\
c \frac{\left|x_{0}\right|}{h\left(t_{0}\right)} \exp \left(\int_{t_{0}}^{t} c \frac{h(s)}{h(\sigma(s))}|F(s)| \Delta s\right) & \text { if } \mu=0\end{cases} \\
& \leq c \frac{\left|x_{0}\right|}{h\left(t_{0}\right)} \exp \left(\int_{t_{0}}^{t} c \frac{h(s)}{h(\sigma(s))}|F(s)| \Delta s\right) \\
& \leq c \frac{\left|x_{0}\right|}{h\left(t_{0}\right)} \exp \left(\int_{t_{0}}^{\infty} c \frac{h(s)}{h(\sigma(s))}|F(s)| \Delta s\right) \\
& \leq c \frac{\left|x_{0}\right|}{h\left(t_{0}\right)} e^{c \beta}
\end{aligned}
$$

for all $t \geq t_{0} \in \mathbb{T}$. Thus,

$$
|x(t)| \leq d\left|x_{0}\right| h(t) h\left(t_{0}\right)^{-1}, \quad t \geq t_{0}
$$

where $d=c e^{c \beta} \geq 1$. Hence, (3.23) is $h$-stable.

Corollary 3.17. Suppose that (3.2) is $h$-stable with bounded $h(t) / h(\sigma(t))$ for each $t \in \mathbb{T}$. Then, (3.23) is $h$-stable if there exists a positive constant $\beta$ such that for all $t_{0} \in \mathbb{T}$,

$$
\int_{t_{0}}^{\infty}|F(s)| \Delta s \leq \beta
$$


Corollary 3.18. When $\mathbb{T}=\mathbb{R},(3.23)$ is h-stable if there exists a positive constant $\beta$ such that for all $t_{0} \in \mathbb{R}$,

$$
\int_{t_{0}}^{\infty}|F(s)| d s \leq \beta
$$

Corollary 3.19. When $\mathbb{T}=\delta \mathbb{Z}$ with a positive constant $\delta$, (3.23) is $h$-stable if there exists a positive constant $\beta$ such that for all $t_{0} \in \delta \mathbb{Z}$,

$$
\sum_{s=t_{0}}^{\infty} \frac{h(s)}{h(s+\delta)}|F(s)| \leq \beta .
$$

We consider the nonlinear perturbed dynamic system

$$
z^{\Delta}(t)=A(t) z(t)+G(t, z(t)),
$$

where $A \in C_{\mathrm{rd}}\left(\mathbb{T}, M_{n}(\mathbb{R})\right)$ and $G$ is rd-continuous on $\mathbb{T} \times \mathbb{R}^{n}$.

We recall the notion of the class $\widehat{H}$.

Definition 3.20. A function $w: \mathbb{R}^{+} \rightarrow \mathbb{R}^{+}$belongs to the class $\widehat{H}$ if

$\left(\mathrm{H}_{1}\right) w(u)$ is nondecreasing and continuous for $u \geq 0$ and positive for $u>0$,

$\left(\mathrm{H}_{2}\right)$ there exists a continuous function $\phi$ on $\mathbb{R}^{+}$with $w(\alpha u) \leq \phi(\alpha) w(u)$ for $\alpha>0, u \geq 0$,

$\left(\mathrm{H}_{3}\right) \lim _{u \rightarrow 0^{+}}(w(u) / u)$ exists.

Theorem 3.21. Suppose that (3.2) is h-stable and

$$
|G(t, x)| \leq F(t) w(|x|), \quad t \geq t_{0},
$$

where $F$ is positive and $r d$-continuous, and $w \in \widehat{H}$ with corresponding multiplier function $\phi$ and

$$
\begin{aligned}
& \lambda(t)=\frac{h\left(t_{0}\right) F(t)}{h(\sigma(t))\left|z_{0}\right|} \phi\left(\frac{\left|z_{0}\right| h(t)}{h\left(t_{0}\right)}\right), \\
& d=W^{-1}\left[W(c)+c \int_{t_{0}}^{\infty} \lambda(s) \Delta s\right]
\end{aligned}
$$

in [13, Theorem 5.8]. Then, (3.36) is h-stable.

Proof. It follows from Lemma 3.15 that the solution $z(t)$ of (3.36) is given by

$$
z(t)=\Phi_{A}\left(t, t_{0}\right) z_{0}+\int_{t_{0}}^{t} \Phi_{A}(t, \sigma(s)) G(s, z(s)) \Delta s, \quad t \geq t_{0},
$$

where $\Phi_{A}\left(t, t_{0}\right)$ is a transition matrix of (3.2).

By $h$-stability of (3.2), there exists a positive bounded function $h(t)$ such that

$$
\left|\Phi_{A}\left(t, t_{0}\right)\right| \leq \operatorname{ch}(t) h\left(t_{0}\right)^{-1}
$$

for all $t \geq t_{0} \in \mathbb{T}$.

The rest of the proof is the same as that of [3, Theorem 2.10]. 
Sung Kyu Choi et al.

Now, we obtain some results about the $h$-stability of system (3.36) in the following corollaries.

Corollary 3.22. Suppose that (3.2) is $h$-stable with a nondecreasing function $h(t)$ and

$$
|G(t, x)| \leq F(t) w(|x|), \quad t \geq t_{0},
$$

where $w \in \widehat{H}$ with corresponding multiplier function $\phi(\alpha)=\alpha$ for all $\alpha>0$ and

$$
d=W^{-1}\left[W(c)+c \int_{t_{0}}^{\infty} F(s) \Delta s\right] .
$$

Then, (3.36) is h-stable.

Corollary 3.23. Suppose that (3.2) is h-stable and

$$
|G(t, x)| \leq F(t) w(|x|), \quad t \geq t_{0} \in \mathbb{T}=\mathbb{R},
$$

where $w \in \widehat{H}$ with corresponding multiplier function $\phi$ and

$$
\begin{aligned}
& \lambda(t)=\frac{h\left(t_{0}\right) F(t)}{h(t)\left|z_{0}\right|} \phi\left(\frac{\left|z_{0}\right| h(t)}{h\left(t_{0}\right)}\right), \\
& d=W^{-1}\left[W(c)+c \int_{t_{0}}^{\infty} \lambda(s) d s\right] .
\end{aligned}
$$

Then, (3.36) is h-stable.

Corollary 3.24. Suppose that (3.2) is h-stable and

$$
|G(t, x)| \leq F(t) w(|x|), \quad t \geq t_{0} \in \mathbb{T}=\delta \mathbb{Z},
$$

where $w \in \widehat{H}$ with corresponding multiplier function $\phi$ and

$$
\begin{aligned}
& \lambda(t)=\frac{h\left(t_{0}\right) F(t)}{h(t+\delta)\left|z_{0}\right|} \phi\left(\frac{\left|z_{0}\right| h(t)}{h\left(t_{0}\right)}\right), \\
& d=W^{-1}\left[W(c)+c \delta \sum_{s \in \delta \mathbb{Z}_{t_{0}}} \lambda(s)\right] .
\end{aligned}
$$

Then, (3.36) is h-stable.

We examine the property of $h$-stability for the perturbed dynamic system (3.24) on time scale $\mathbb{T}$.

Lemma 3.25. Suppose that $k \in C_{\mathrm{rd}}\left(\mathbb{T} \times \mathbb{R}_{+}, \mathbb{R}\right)$ is nondecreasing in the second argument $x$ for each fixed $t \geq t_{0}$ with the property

$$
x(t)-\int_{t_{0}}^{t} k(s, x(s)) \Delta s \leq y(t)-\int_{t_{0}}^{t} k(s, y(s)) \Delta s, \quad t \geq t_{0} \in \mathbb{T},
$$

for $x, y \in C_{\mathrm{rd}}\left(\mathbb{T}, \mathbb{R}_{+}\right)$. If $x\left(t_{0}\right)<y\left(t_{0}\right)$, then $x(t)<y(t)$ for all $t \geq t_{0} \in \mathbb{T}$. 
Proof. Suppose that there exists a $\tau \in \mathbb{T}$ with $t_{0}<\tau$ such that $x(s)<y(s)$ for $t_{0} \leq s<\tau$ and $x(\tau) \geq y(\tau)$. Also, we obtain

$$
x(\tau)-\int_{t_{0}}^{\tau} k(s, x(s)) \Delta s>y(\tau)-\int_{t_{0}}^{\tau} k(s, y(s)) \Delta s, \quad \tau \geq t_{0} \in \mathbb{T} .
$$

This is a contradiction.

If $\mathbb{T}=\mathbb{R}$ and $\mathbb{T}=\delta \mathbb{Z}$ with a positive constant $\delta$, then we can obtain the following results as corollaries of Lemma 3.25.

Corollary 3.26 (see [14, Lemma 2.1]). Suppose that $k \in C\left(\mathbb{R} \times \mathbb{R}_{+}, \mathbb{R}\right)$ is nondecreasing in the second argument $x$ for $t \geq t_{0}$ with the property

$$
x(t)-\int_{t_{0}}^{t} k(s, x(s)) d s \leq y(t)-\int_{t_{0}}^{t} k(s, y(s)) d s, \quad t \geq t_{0} \in \mathbb{R},
$$

for $x, y \in C\left(\mathbb{T}, \mathbb{R}_{+}\right)$. If $x\left(t_{0}\right)<y\left(t_{0}\right)$, then $x(t)<y(t)$ for all $t \geq t_{0} \in \mathbb{R}$.

Corollary 3.27 (see [5, Lemma 9]). Suppose that $k: \delta \mathbb{Z} \times \mathbb{R}_{+} \rightarrow \mathbb{R}$ is nondecreasing in the second argument $x$ for $t \geq t_{0}$ with the property

$$
x(t)-\sum_{s=t_{0}}^{t-\delta} k(s, x(s)) \delta \leq y(t)-\sum_{s=t_{0}}^{t-\delta} k(s, y(s)) \delta, \quad t \geq t_{0} \in \delta \mathbb{Z},
$$

for $x, y: \delta \mathbb{Z} \rightarrow \mathbb{R}_{+}$. If $x\left(t_{0}\right)<y\left(t_{0}\right)$, then $x(t)<y(t)$ for all $t \geq t_{0} \in \delta \mathbb{Z}$.

Theorem 3.28. Assume that $x=0$ of (3.2) is uniformly stable. Suppose that

$$
|g(t, x)| \leq \iota(t,|x|),
$$

where $\iota \in C_{\mathrm{rd}}\left(\mathbb{T} \times \mathbb{R}_{+}, \mathbb{R}_{+}\right)$is strictly increasing in $u$ for each fixed $t \in \mathbb{T}_{t_{0}}$ with $\iota(t, 0)=0$. Consider the scalar dynamic equation

$$
u^{\Delta}=c l(t, u), \quad u\left(t_{0}\right)=u_{0}, \quad t \in \mathbb{T}_{t_{0}},
$$

where $c \geq 1$ is a constant. If $u=0$ of (3.52) is h-stable, then (3.24) is h-stable whenever $u_{0}=c\left|x_{0}\right|$.

Proof. It follows from Lemma 3.15 that the solution $x(t)$ of (3.24) is given by

$$
x(t)=\Phi_{A}\left(t, t_{0}\right) x_{0}+\int_{t_{0}}^{t} \Phi_{A}(t, \sigma(s)) g(s, x(s)) \Delta s, \quad t \in \mathbb{T}_{t_{0}} .
$$

Since $x=0$ of (3.2) is uniformly stable, we obtain

$$
|x(t)| \leq\left|\Phi_{A}\left(t, t_{0}\right)\right|\left|x_{0}\right|+\int_{t_{0}}^{t}\left|\Phi_{A}(t, \sigma(s))\right||g(s, x(s))| \Delta s \leq c\left|x_{0}\right|+c \int_{t_{0}}^{t} \iota(s,|x(s)|) \Delta s .
$$

Thus, we have

$$
|x(t)|-c \int_{t_{0}}^{t} \iota(s,|x(s)|) \Delta s \leq c\left|x_{0}\right| \leq u_{0}=u(t)-c \int_{t_{0}}^{t} \iota(s, u(s)) \Delta s, \quad t \in \mathbb{T}_{t_{0}} .
$$


By Lemma 3.25, we have $|x(t)|<u(t)$ for all $t \in \mathbb{T}_{t_{0}}$. Since $u=0$ of (3.52) is $h$-stable, we obtain

$$
|x(t)| \leq c_{1} u_{0} h(t) h\left(t_{0}\right)^{-1} \leq c_{1} c\left|x_{0}\right| h(t) h\left(t_{0}\right)^{-1}=M\left|x_{0}\right| h(t) h\left(t_{0}\right)^{-1}, \quad t \in \mathbb{T}_{t_{0}}
$$

where $M=c_{1} c \geq 1$ is a constant. This completes the proof.

\section{Acknowledgment}

This work was supported by the Korea Research Foundation Grant founded by Korea Government (MOEHRD, KRF-2005-070-C00015).

\section{References}

[1] S. Hilger, "Analysis on measure chains—a unified approach to continuous and discrete calculus," Results in Mathematics, vol. 18, no. 1-2, pp. 18-56, 1990.

[2] M. Bohner and A. Peterson, Dynamic Equations on Time Scales: An Introduction with Applications, Birkhäuser, Boston, Mass, USA, 2001.

[3] S. K. Choi, N. J. Koo, and D. M. Im, “h-stability for linear dynamic equations on time scales," Journal of Mathematical Analysis and Applications, vol. 324, no. 1, pp. 707-720, 2006.

[4] M. Pinto, "Perturbations of asymptotically stable differential systems," Analysis, vol. 4, no. 1-2, pp. $161-175,1984$.

[5] S. K. Choi and N. J. Koo, "Variationally stable difference systems by $n_{\infty}$-similarity," Journal of Mathematical Analysis and Applications, vol. 249, no. 2, pp. 553-568, 2000.

[6] S. K. Choi, N. J. Koo, and H. S. Ryu, "h-stability of differential systems via $t_{\infty}$-similarity," Bulletin of the Korean Mathematical Society, vol. 34, no. 3, pp. 371-383, 1997.

[7] M. Pinto, "Integral inequalities of Bihari-type and applications," Funkcialaj Ekvacioj, vol. 33, no. 3, pp. 387-403, 1990.

[8] R. J. Marks II, I. A. Gravagne, J. M. Davis, and J. J. DaCunha, "Nonregressivity in switched linear circuits and mechanical systems," Mathematical and Computer Modelling, vol. 43, no. 11-12, pp. 13831392, 2006.

[9] R. Agarwal, M. Bohner, D. O'Regan, and A. Peterson, "Dynamic equations on time scales: a survey," Journal of Computational and Applied Mathematics, vol. 141, no. 1-2, pp. 1-26, 2002.

[10] V. Lakshmikantham, S. Sivasundaram, and B. Kaymakcalan, Dynamic Equations on Measure Chains, Kluwer Academic Publishers, Dordrecht, The Netherlands, 1996.

[11] C. Pötzsche, Langsame Faserbünder dynamischer Gleichungen auf Maßketten, Ph.D. thesis, Universität Augsburg, Augsburg, Germany, 2002.

[12] J. J. DaCunha, "Stability for time varying linear dynamic systems on time scales," Journal of Computational and Applied Mathematics, vol. 176, no. 2, pp. 381-410, 2005.

[13] R. Agarwal, M. Bohner, and A. Peterson, "Inequalities on time scales: a survey," Mathematical Inequalities E Applications, vol. 4, no. 4, pp. 535-557, 2001.

[14] S. K. Choi and N. J. Koo, "h-stability for nonlinear perturbed systems," Annals of Differential Equations, vol. 11, no. 1, pp. 1-9, 1995. 


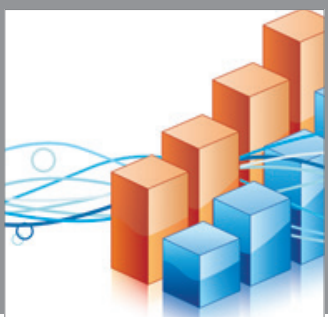

Advances in

Operations Research

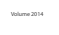

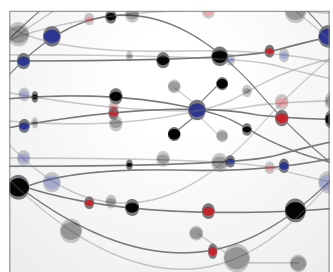

\section{The Scientific} World Journal
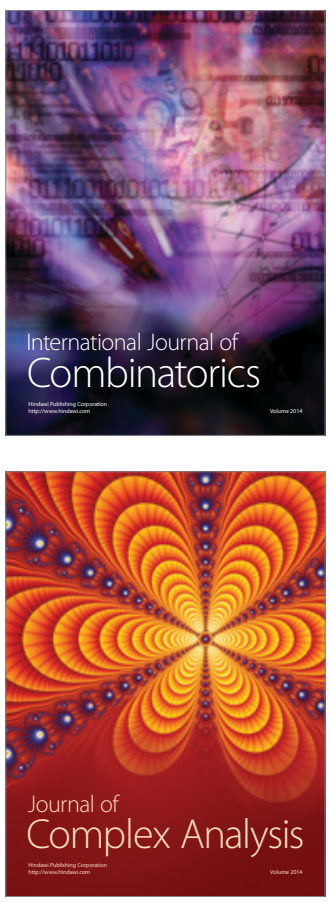

International Journal of

Mathematics and

Mathematical

Sciences
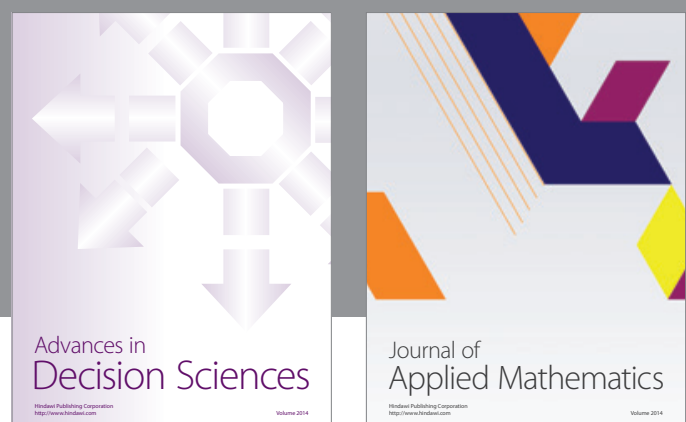

Journal of

Applied Mathematics
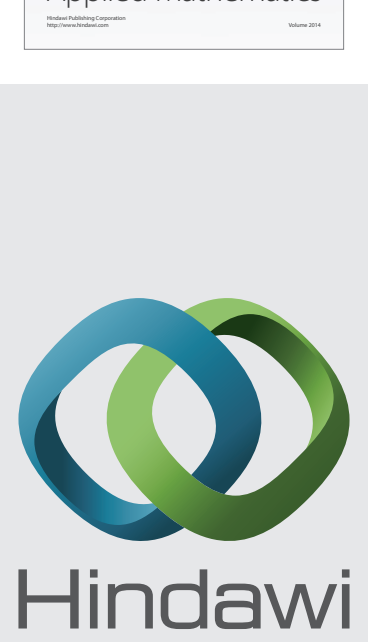

Submit your manuscripts at http://www.hindawi.com
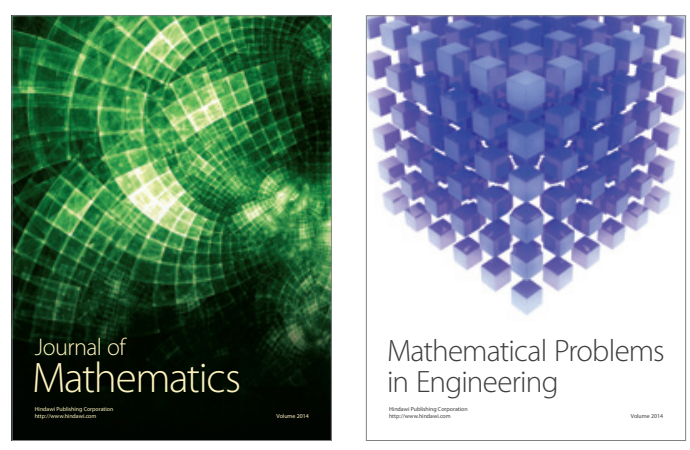

Mathematical Problems in Engineering
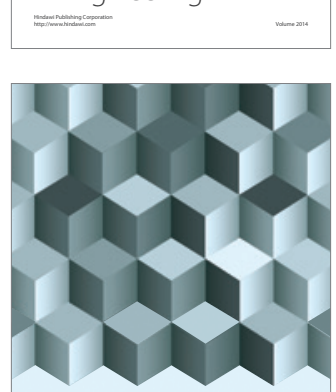

Journal of

Function Spaces
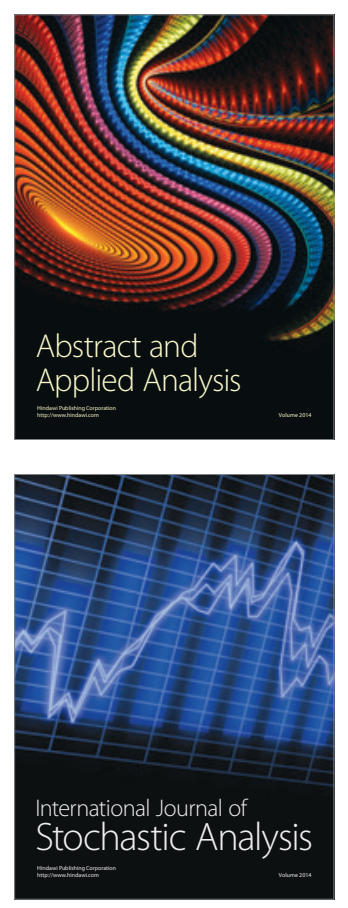

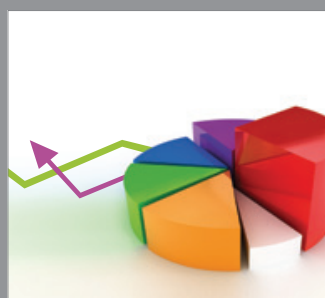

ournal of

Probability and Statistics

Promensencen
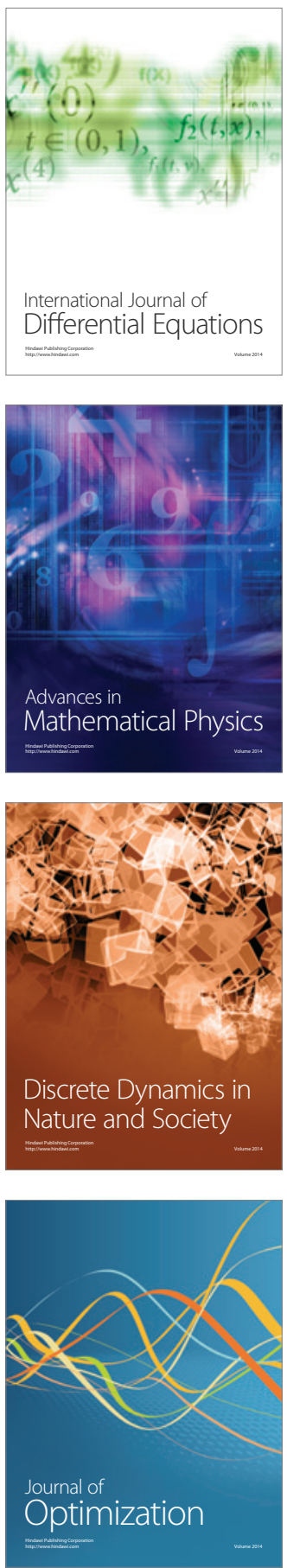